\title{
Georg schmorl prize of the German spine society (DWG) 2021: Spinal Instability Spondylodiscitis Score (SISS) - a novel classification system for spinal instability in spontaneous spondylodiscitis
}

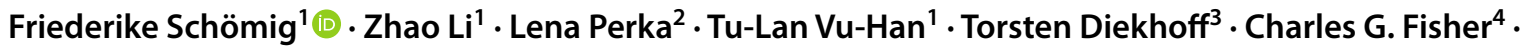 \\ Matthias Pumberger ${ }^{1}$
}

Received: 16 January 2022 / Revised: 16 January 2022 / Accepted: 14 February 2022 / Published online: 7 March 2022

(c) The Author(s) 2022

\begin{abstract}
Purpose Even though spinal infections are associated with high mortality and morbidity, their therapy remains challenging due to a lack of established classification systems and widely accepted guidelines for surgical treatment. This study's aim therefore was to propose a comprehensive classification system for spinal instability based on the Spinal Instability Neoplastic Score (SINS) aiding spine surgeons in choosing optimal treatment for spontaneous spondylodiscitis.

Methods Patients who were treated for spontaneous spondylodiscitis and received computed tomography (CT) imaging were included retrospectively. The Spinal Instability Spondylodiscitis Score (SISS) was developed by expert consensus. SINS and SISS were scored in CT-images by four readers. Intraclass correlation coefficients (ICCs) and Fleiss' Kappa were calculated to determine interrater reliabilities. Predictive validity was analyzed by cross-tabulation analysis.

Results A total of 127 patients were included, 94 (74.0\%) of which were treated surgically. Mean SINS was $8.3 \pm 3.2$, mean SISS 8.1 \pm 2.4 . ICCs were 0.961 (95\%-CI: 0.949-0.971) for total SINS and 0.960 (95\%-CI: 0.946-0.970) for total SISS. SINS yielded false positive and negative rates of $12.5 \%$ and $67.6 \%$, SISS of $15.2 \%$ and $40.0 \%$, respectively.

Conclusion We show high reliability and validity of the newly developed SISS in detecting unstable spinal lesions in spontaneous spondylodiscitis. Therefore, we recommend its use in evaluating treatment choices based on spinal biomechanics. It is, however, important to note that stability is merely one of multiple components in making surgical treatment decisions.
\end{abstract}

Keywords Discitis · Infections · Classification · Spine

Friederike Schömig

friederike.schoemig@charite.de

Zhao Li

zhao.li@charite.de

Lena Perka

lena.perka@stud.pmu.ac.at

Tu-Lan Vu-Han

tu-lan.vu-han@charite.de

Torsten Diekhoff

torsten.diekhoff@charite.de

Charles G. Fisher

charles.fisher@vch.ca

Matthias Pumberger

matthias.pumberger@charite.de
1 Centrum Für Muskuloskeletale Chirurgie, Charité Universitätsmedizin Berlin, Charitéplatz 1, 10117 Berlin, Germany

2 Salzburg - Universitätsklinikum der Paracelsus Medizinischen Privatuniversität, Müllner Hauptstraße 48, 5020 Salzburg, Austria

3 Klinik Für Radiologie, Charité - Universitätsmedizin Berlin, Charitéplatz 1, 10117 Berlin, Germany

4 Division of Spine Surgery, University of British Columbia and Vancouver General Hospital, 818 West 10th Avenue, Vancouver, BC, Canada 


\section{Introduction}

Infections of the spine account for 2-7\% of all musculoskeletal infections $[1,2]$. Even though they are associated with significant mortality and morbidity, there remains no established classification systems and treatment algorithms to optimize management. While first-line treatment usually is conservative via intravenous antibiotics, surgical treatment is generally warranted for source control, epidural abscess, neurological deficits, or spinal instability [3]. Thus, using a multidisciplinary team of infectious disease physicians, interventional radiologists, and spine surgeons to best employ proper treatment and timing of treatment is critical.

While most patients with spondylodiscitis should receive surgical consultation, there are no widely accepted guidelines for surgical treatment decisions. Even though instability has been identified as a common indication for surgery, there still is a lack of evidence-based criteria to define spinal instability in spondylodiscitis [4]. Spine surgeons therefore need to rely on clinical experience and various ill-defined radiographic features rather than objective criteria in their surgical decision-making process. Furthermore, there is no common language allowing for appropriate and timely referrals of spondylodiscitis patients from internists, radiologists or infectious disease specialists to spine surgeons.

In the setting of spinal metastasis, the Spine Oncology Study Group (SOSG) defined spine instability as "the loss of spinal integrity as a result of a neoplastic process that is associated with movement-related pain, symptomatic or progressive deformity, and/or neural compromise under physiological loads." [5] Based on this definition, best available evidence, and a Delphi technique, the SOSG developed the Spinal Instability Neoplastic Score (SINS), a classification system with radiographic and patient factors for diagnosing neoplastic spinal instability. Depending on the total score, the spinal lesion is defined as stable, potentially unstable, or unstable. In case of a potentially unstable or unstable lesion, referral to a spine surgeon for evaluation of the need for surgical intervention is recommended [5, 6]. The SINS has greatly facilitated referral across surgical and oncology disciplines and heightened awareness of neoplastic spinal instability [7, 8].

Similar to metastatic spinal lesions, spinal infection causes instability over a period of time which therefore is different from instability caused by traumatic injury. Despite a recent study by Pithwa et al. that found extrapolation of the SINS toward the evaluation of instability in spinal tuberculosis to be useful, certain parameters of the SINS do not apply in spinal infection [9]. Therefore, the purpose of this study was to propose and psychometrically evaluate a classification system based on the widely accepted SINS to aid physicians and surgeons to determine instability in patients with spondylodiscitis to not only facilitate referral between disciplines but also to aid in surgical decision-making.

\section{Methods}

\section{Patients and ethical approval}

The study was approved by the institutional ethics committee (EA1/019/21). Patients who were treated for spondylodiscitis at our department between January 2006 and December 2020 were included retrospectively. Exclusion criteria were previous interventions at the same or adjacent spinal levels and incomplete clinical or radiological data. Patients were identified using information from discharge letters and diagnostic and procedural codes, i.e., diagnostic related groups (DRG) codes. Clinical data were retrieved from electronic medical reports and patient charts including demographic parameters (age, gender), body mass index (BMI), pain at admission, and the performed treatment. Pain was assessed by numeric rating scale (NRS) scores. Treatment was conservative unless there was present or imminent neurological deficit and/or present or imminent spinal instability.

Spondylodiscitis was defined by a combination of characteristic radiological changes of the intervertebral disc in magnetic resonance imaging (MRI) and/or computed tomography $(\mathrm{CT})$ scans and clinical findings including elevated CRP levels, elevated WBC, back and/or neck pain as well as fever. Localization of the infection was classified into three regions: cervical, thoracic, or lumbar. Spondylodiscitis of the cervicothoracic junction $(\mathrm{C} 7 / \mathrm{Th} 1)$ was counted as cervical, the thoracolumbar junction (Th12/L1) as thoracic and the lumbosacral junction (L5/S1) as lumbar spondylodiscitis.

\section{Image analysis}

Three readers (reader 1, an orthopaedic surgeon with eleven years of experience; reader 2 , an orthopaedic surgery resident with three years of experience; reader 3 , an orthopaedic surgery resident with two years of experience) independently evaluated CT-images. A two-out-of-three-reader agreement approach was used for all scored parameters. Further disagreement was solved by a radiologist specializing in musculoskeletal diseases with eleven years of experience. For calculation of interrater reliability, a fourth reader (a medical student trained in musculoskeletal imaging) additionally evaluated CT-images. 
Table 1 Parameters of the Spinal Instability Neoplastic Score (SINS) as previously published by Fisher et al. [5]

\begin{tabular}{|c|c|}
\hline Parameter & Score \\
\hline \multicolumn{2}{|l|}{ Location } \\
\hline Junctional (occiput-C2, C7-T2, T11-L1, L5-S1) & 3 \\
\hline Mobile Spine (C3-6, L2-4) & 2 \\
\hline Semirigid (T3-10) & 1 \\
\hline Rigid (S2-5) & 0 \\
\hline \multicolumn{2}{|l|}{ Bone lesion } \\
\hline Lytic & 2 \\
\hline Mixed (lytic/blastic) & 1 \\
\hline Blastic & 0 \\
\hline \multicolumn{2}{|l|}{ Spinal alignment } \\
\hline Subluxation/Translation & 4 \\
\hline De novo deformity (kyphosis/scoliosis) & 2 \\
\hline Normal alignment & 0 \\
\hline \multicolumn{2}{|l|}{ Vertebral body collapse } \\
\hline$>50 \%$ collapse & 3 \\
\hline$<50 \%$ collapse & 2 \\
\hline No collapse with $>50 \%$ body involved & 1 \\
\hline None of the above & 0 \\
\hline \multicolumn{2}{|l|}{ Posterolateral involvement of the spinal elements } \\
\hline Bilateral & 3 \\
\hline Unilateral & 1 \\
\hline None of the above & 0 \\
\hline \multicolumn{2}{|l|}{ Mechanical pain } \\
\hline Yes & 3 \\
\hline Occasional pain but not mechanical & 1 \\
\hline Pain-free lesion & 0 \\
\hline \multicolumn{2}{|l|}{ Total score } \\
\hline $0-6$ & Stable lesion \\
\hline $7-12$ & Potentially unstable lesion \\
\hline $13-18$ & Unstable lesion \\
\hline
\end{tabular}

\section{Spinal Instability Neoplastic Score}

SINS was scored as previously published by Fisher et al. (Table 1) [5]. Posterior column disintegration was defined as facet disruption, fractures at the junction of pedicle and vertebral body, or osteolysis of one or both pedicles. A total score of 0-6 was defined as stable, 7-12 as potentially unstable, and 13-18 as unstable.

\section{Spinal Instability Spondylodiscitis Score}

For the Spinal Instability Spondylodiscitis Score (SISS), a new granular scoring systems was developed after a review of the existing literature by expert consensus of two orthopaedic surgeons based on the parameters of the SINS. This new classification system includes four parameters which are presented in Table 2. A total score of 0-4 was defined as stable, 5-9 as potentially unstable, and 10-14 as unstable spondylodiscitis.

\section{Statistical analysis}

For determination of interrater reliability between the four readers, two-way random single-measure intraclass correlation coefficients (ICCs) were calculated to measure interrater agreement for total SINS and SISS scores and Fleiss' Kappa for multiple readers was calculated for each of the components of the SINS and SISS. Kappa values of $<0.00$ were rated as poor, 0.00-0.20 as slight, 0.21-0.40 as fair, $0.41-0.60$ as moderate, $0.61-0.80$ as substantial, and $0.81-1.00$ as almost perfect agreement [10]. Predictive validity was analyzed by cross-tabulation analysis. The statistical significance level for all tests performed was $\mathrm{p}<0.05$. Statistical analysis was performed using SPSS version 27 (SPSS Inc., Chicago, Illinois). 
Table 2 Parameters of the Spinal Instability Spondylodiscitis Score (SISS)

\begin{tabular}{ll}
\hline Parameter & Score \\
\hline Location & \\
Junctional (occiput-C2, C7-T2, T11-L1, & 3 \\
$\quad$ L5-S1) & 2 \\
Mobile Spine (C3-6, L2-4) & 1 \\
Semirigid (T3-10) & 0 \\
Rigid (S2-5) & \\
Bone lesion & 4 \\
$>$ 50\% vertebral body involvement & 2 \\
$<50 \%$ vertebral body involvement & 1 \\
Endplate involvement & 0 \\
Intact endplates & \\
Spinal alignment & 4 \\
Subluxation/Translation & 2 \\
De novo deformity (kyphosis/scoliosis) & 0 \\
Normal alignment & \\
Mechanical pain & \\
Yes & 3 \\
Occasional pain but not mechanical & 1 \\
Pain-free lesion & 0 \\
Total Score & \\
0-4 & Potentially unstable lesion \\
5-9 & \\
10-14 & \\
\hline
\end{tabular}

\section{Results}

\section{Patients}

A total of 247 patients with spondylodiscitis were identified. Fifty-four patients were excluded due to previous interventions at the same or adjacent spinal segments. Eight patients were excluded due to missing clinical data. In 127 of the remaining 185 patients, CT imaging was performed. Fifty-nine (46.5\%) females were included. Mean age was $66.2 \pm 13.2$ years. Nine $(7.1 \%)$ patients had spondylodiscitis of the cervical spine, 47 (36.4\%) of the thoracic spine, and 71 (56.6\%) of the lumbar spine. Demographic and clinical data of included patients are presented in Table 3.

\section{Interobserver Reliability}

For SINS, Fleiss' Kappa was $0.413(p<0.001)$ for bone lesion, $0.588(p<0.001)$ for spinal alignment, 0.514 $(p<0.001)$ for vertebral body collapse, and $0.520(p<0.001)$ for posterolateral involvement. ICC for total SINS was 0.961 (95\% CI: 0.949-0.971).
Table 3 Demographic parameters of included patients presenting with spontaneous spondylodiscitis. Mean values are given with standard deviation

\begin{tabular}{ll}
\hline & Patients $(n=127)$ \\
\hline Age (years) & $66.1 \pm 13.4$ \\
Sex (f:m) & $59: 68$ \\
BMI (kg/m $\left.{ }^{2}\right)$ & $26.9 \pm 6.0$ \\
Symptoms & \\
Low back pain resting (VAS) & $3.9 \pm 2.6$ \\
Low back pain moving (VAS) & $5.6 \pm 2.8$ \\
Localization & \\
Cervical & $9(7.1 \%)$ \\
Thoracic & $47(37.0 \%)$ \\
Lumbar & $71(55.9 \%)$ \\
Treatment & \\
Conservative & $33(26.0 \%)$ \\
Surgical & $94(74.0 \%)$ \\
\hline
\end{tabular}

For SISS, Fleiss' Kappa was $0.598(p<0.001)$ for bone lesion and $0.588(p<0.001)$ for spinal alignment. ICC for total SISS was 0.960 (95\% CI: 0.946-0.970).

\section{Validity}

Imaging examples of two patients are given in Fig. 1.

Mean SINS was $8.3 \pm 3.2$. Table 4 shows scores determined by consensus and the performed treatment. By consensus, $37(29.1 \%)$ cases were defined as stable, $74(5.8 \%)$ as potentially unstable, and $16(12.6 \%)$ as unstable lesions. Twenty-five patients were treated surgically despite being classified as stable. Two patients were treated conservatively despite being classified as unstable. This yields a false positive rate (unstable lesion with conservative treatment) of $12.5 \%$ and false negative rate of $67.6 \%$ (stable lesion with surgical treatment).

Mean SISS was $8.1 \pm 2.4$. Table 5 shows scores determined by consensus and the performed treatment. By consensus, $10(7.9 \%)$ cases were defined as stable, $84(66.1 \%)$ as potentially unstable, and $33(26.0 \%)$ as unstable spondylodiscitis. Four patients were treated surgically despite being classified as stable due to progressive neurological symptoms caused by epidural abscess formation. Five patients were treated conservatively despite being classified as unstable. In two of these cases, patient factors such as multiple concomitant diseases or patient refusal led to this decision. In three cases, spinal deformity was found to be caused by degeneration or inflammatory diseases rather than infection. This yields a false positive rate of $15.2 \%$ (unstable lesion with conservative treatment) and a false negative rate of $40.0 \%$ (stable lesion with surgical treatment). 
Fig. 1 a Sagittal and coronal CT-scans of a 71-year-old male patient with spondylodiscitis Th7-9. Both SINS and SISS consensus scorings were consistent with an unstable lesion. The patient was treated surgically by posterior fusion Th5/7-10/11. b Sagittal and coronal CT-scans of a 63-yearold male patient with spondylodiscitis Th9/10. While SINS consensus scoring yielded a stable lesion, SISS consensus scoring was consistent with an unstable lesion. The patient was treated surgically by posterior fusion Th8-11
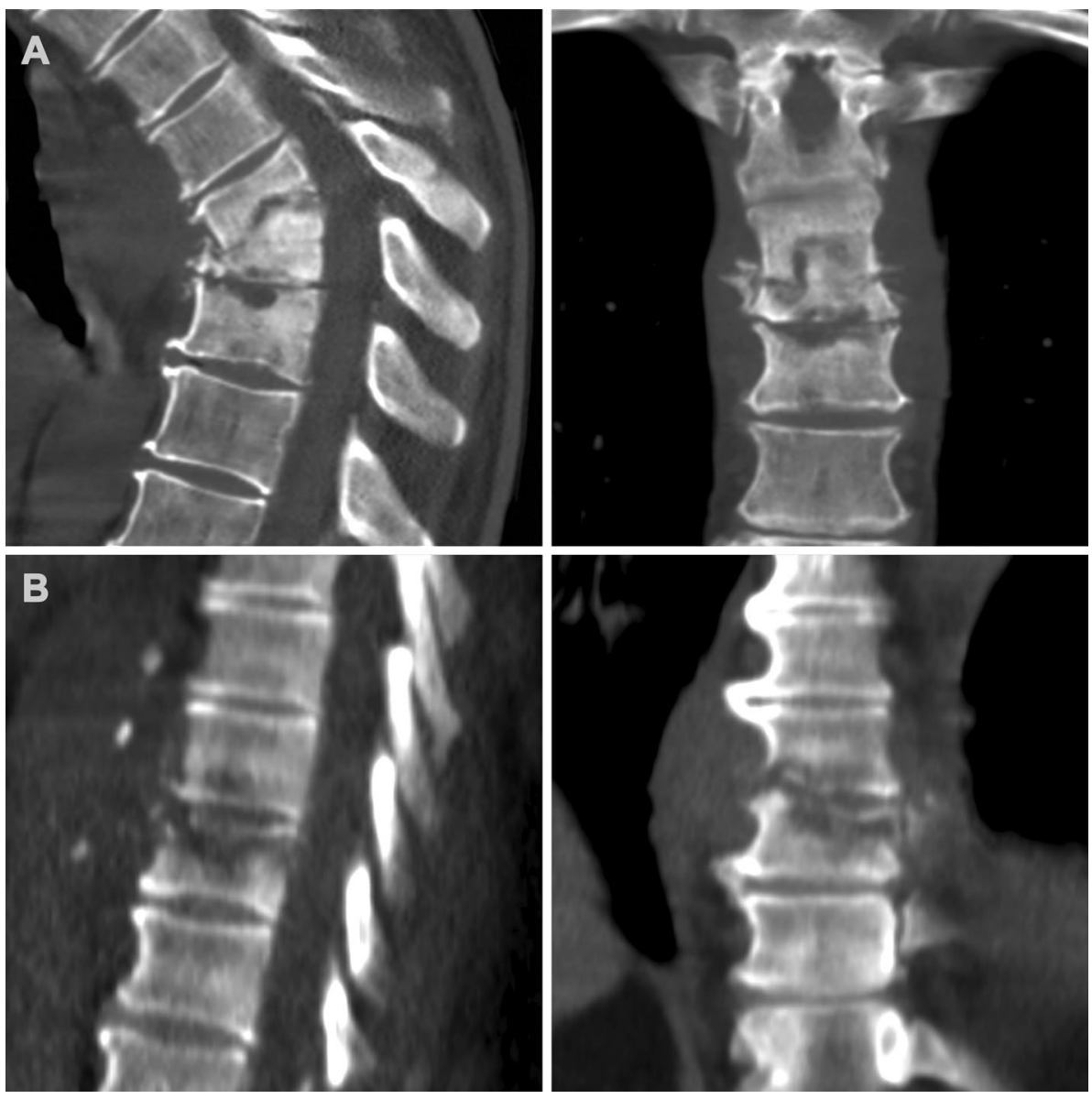

Table 4 Cross-tabulation of total Spinal Instability Neoplastic Score (SINS) and performed treatment

\begin{tabular}{llll}
\hline SINS & Surgical treatment & $\begin{array}{l}\text { Conservative treat- } \\
\text { ment }\end{array}$ & Total \\
\hline 2 & 1 & 0 & 1 \\
3 & 2 & 2 & 4 \\
4 & 5 & 5 & 10 \\
5 & 9 & 2 & 11 \\
6 & 8 & 3 & 11 \\
7 & 11 & 6 & 17 \\
8 & 15 & 3 & 18 \\
9 & 10 & 7 & 17 \\
10 & 12 & 1 & 13 \\
11 & 3 & 2 & 5 \\
12 & 4 & 0 & 4 \\
13 & 6 & 0 & 6 \\
14 & 3 & 0 & 3 \\
15 & 1 & 2 & 3 \\
16 & 4 & 0 & 4 \\
Total & 94 & 33 & 127 \\
\hline
\end{tabular}

Table 5 Cross-tabulation of total Spinal Instability Spondylodiscitis Score (SISS) and performed treatment

\begin{tabular}{llll}
\hline SISS & Surgical treatment & $\begin{array}{l}\text { Conservative treat- } \\
\text { ment }\end{array}$ & Total \\
\hline 2 & 1 & 0 & 1 \\
3 & 1 & 0 & 1 \\
4 & 2 & 6 & 8 \\
5 & 8 & 3 & 11 \\
6 & 8 & 4 & 12 \\
7 & 15 & 3 & 18 \\
8 & 19 & 4 & 23 \\
9 & 12 & 8 & 20 \\
10 & 11 & 0 & 11 \\
11 & 10 & 3 & 13 \\
12 & 3 & 0 & 3 \\
13 & 4 & 1 & 5 \\
14 & 0 & 1 & 1 \\
Total & 94 & 33 & 127 \\
\hline
\end{tabular}




\section{Discussion}

This study proposes a new classification system providing objective criteria for evaluating spinal stability in spontaneous spondylodiscitis. While instability is only one criterion for the determination of treatment in patients with spinal infections, our results show that it plays a crucial role in the clinical decision-making process as it accurately predicts the chosen treatment. Thus, the proposed classification system is the first to aid physicians in determining whether surgical referral is necessary and spine surgeons in deciding whether surgical treatment is indicated in spinal infections based on biomechanics.

Spinal instability is defined as the ability of the spine to maintain its functionality while preventing neurologic deficit, pain, and abnormal angulation [11]. Similar to spinal instability caused by neoplastic lesions, instability associated with spinal infection appears to be a clinical entity based on symptoms and imaging that is different from instability caused by traumatic injuries as it develops over a period of time rather than acutely [5]. Thus, we adapted the scoring system developed by the SOSG for metastatic spinal lesions to better represent typical findings in spondylodiscitis. According to this new classification system, instability caused by spondylodiscitis is associated with localization, mechanical pain, spinal deformity, and vertebral body affection. Localization, mechanical pain, and spinal deformity were adopted from the SINS as we found them to be well-suited to describe instability caused by infection: Junctional regions of the spine are at higher risk for instability causing deformity, which is why as in oncologic lesions, they received the highest score in the newly developed SISS. Rigid segments, in contrast, are biomechanically protected and therefore received the lowest score. As reported for oncologic instability, mechanical neck or back pain is a typical symptom of infectious spinal diseases [12]. This type of pain is associated with structural abnormality of the spine, which is why we included it in the SISS as well [5]. Spinal deformity has previously been described as indicating instability by several authors [13-15]. As kyphosis or scoliosis may be compensated and therefore remain stable, they receive less points than subluxations or dislocations. The differentiation of lesions into blastic or lytic lesions does not apply for infectious lesions which is why this parameter was not included in the SISS. Infectious lesions are more comparable to lytic lesions, which as previously described, inherit a greater risk of vertebral body collapse. Thus, a new parameter describing the extent of infectious bone lesions and the accompanying vertebral destruction was developed as it has been shown that higher cross-sectional defect area and bone mineral density correlate with vertebral body failure and pathologic fracture risk [16-18]. This new parameter "vertebral body involvement" therefore receives more weighted points in the SISS than vertebral body collapse does in the SINS. As in the SINS, in case of multiple lesions, scores are not summed, but each lesion needs to be considered separately.

Overall, our results show that grading instability caused by spondylodiscitis according to the newly developed SISS leads to high correlation to the chosen type of treatment with fewer falsely considered stable lesions compared with the SINS. Therefore, SISS is more appropriate in detecting unstable lesions which require surgical treatment. In four cases, surgical treatment was performed even though according to the SISS, conservative management was proposed. All four of these cases did, however, show progressive neurological symptoms due to epidural abscess formation, which is an absolute indication for surgical treatment. In five cases, surgical treatment was not performed even though suggested by the SISS. In two of these cases, patient factors such as multiple concomitant diseases or patient refusal led to this decision. In three cases, spinal deformity was found to be caused by degeneration or inflammation rather than infection, which is why the affected segment was in fact thought to be stable. These cases show that spinal stability is not the only factor that needs to be accounted for in choosing optimal treatment of spondylodiscitis but factors including patient overall health, neurological status, and patient choice need to be considered as well.

Important factors in developing a new classification system are its reproducibility and reliability, but most importantly its ability to comprehensively guide clinical decisionmaking and at the same time being easy to use. Our study shows moderate interrater reliability for all of the SISS parameters and excellent interrater reliability for overall SISS score. This is in concordance with our and previous findings of interrater reliability of the SINS parameters and total SINS scores [6].

Some limitations need to be discussed. While the SISS was developed in an expert consensus process based on the SINS, in contrast to SINS development, only two surgeons were involved in the process and the Delphi technique was not used, which may have caused bias. Furthermore, due to the retrospective design of our study, inherent limitations and bias were present. Even though treatment was performed according to current standards, there was no predefined treatment algorithm, leaving the decision up to the surgeon. Thus, our results need to be validated in a prospective setting. Furthermore, eight cases had to be excluded due to missing imaging or clinical data, which may have caused unknown bias.

In conclusion, we propose a new comprehensive classification system to aid physicians and spine surgeons define spinal instability in spontaneous spondylodiscitis. We show 
similar reliability of this new SISS compared with the widely accepted SINS and high validity especially in detecting unstable lesions which require surgical treatment. We therefore recommend its use in determining whether surgical consultation is necessary and in evaluating treatment choices based on spinal biomechanics. However, it is important to note that stability is merely one of multiple components in making surgical treatment decisions. Patient overall health, neurological status, and patient choice need to be considered as well.

Authors' contributions Study conception and design were developed by Friederike Schömig and Matthias Pumberger. Data collection and analysis were performed by Friederike Schömig, Zhao Li, Lena Perka, Tu-Lan Vu-Han, Torsten Diekhoff, Charles Fisher, and Matthias Pumberger. The first draft of the manuscript was written by Friederike Schömig and all authors commented on previous versions of the manuscript. All authors read and approved the final manuscript.

Funding Open Access funding enabled and organized by Projekt DEAL. The authors did not receive support from any organization for the submitted work.

Data availability The datasets generated during and/or analyzed during the current study are not publicly available due to patient privacy but are available from the corresponding author on reasonable request.

\section{Declarations}

Conflicts of interest The authors have no relevant financial or nonfinancial interests to disclose.

Ethics approval This retrospective chart review study involving human participants was in accordance with the ethical standards of the institutional and national research committee and with the 1964 Declaration of Helsinki and its later amendments or comparable ethical standards. The Human Investigation Committee (IRB) of Charité - Universitätsmedizin Berlin approved this study (EA1/019/21).

Open Access This article is licensed under a Creative Commons Attribution 4.0 International License, which permits use, sharing, adaptation, distribution and reproduction in any medium or format, as long as you give appropriate credit to the original author(s) and the source, provide a link to the Creative Commons licence, and indicate if changes were made. The images or other third party material in this article are included in the article's Creative Commons licence, unless indicated otherwise in a credit line to the material. If material is not included in the article's Creative Commons licence and your intended use is not permitted by statutory regulation or exceeds the permitted use, you will need to obtain permission directly from the copyright holder. To view a copy of this licence, visit http://creativecommons.org/licenses/by/4.0/.

\section{References}

1. Butler JS, Shelly MJ, Timlin M, Powderly WG, O'Byrne JM (2006) Nontuberculous pyogenic spinal infection in adults: a 12-year experience from a tertiary referral center. Spine (Phila Pa 1976) 31:26952700. https://doi.org/10.1097/01.brs.0000244662.78725.37
2. Tyrrell PN, Cassar-Pullicino VN, McCall IW (1999) Spinal infection. Eur Radiol 9:1066-1077. https://doi.org/10.1007/s003300050 793

3. Lang S, Rupp M, Hanses F, Neumann C, Loibl M, Alt V (2021) Infections of the spine : Pyogenic spondylodiscitis and implant-associated vertebral osteomyelitis. Unfallchirurg 124:489-504. https:// doi.org/10.1007/s00113-021-01002-w

4. Fisahn C, Alonso F, Hasan GA, Tubbs RS, Dettori JR, Schildhauer TA, Rustagi T (2017) Trends in spinal surgery for Pott's disease (2000-2016): an overview and bibliometric study. Global Spine J 7:821-828. https://doi.org/10.1177/2192568217735827

5. Fisher CG, DiPaola CP, Ryken TC, Bilsky MH, Shaffrey CI, Berven SH, Harrop JS, Fehlings MG, Boriani S, Chou D, Schmidt MH, Polly DW, Biagini R, Burch S, Dekutoski MB, Ganju A, Gerszten PC, Gokaslan ZL, Groff MW, Liebsch NJ, Mendel E, Okuno SH, Patel S, Rhines LD, Rose PS, Sciubba DM, Sundaresan N, Tomita K, Varga PP, Vialle LR, Vrionis FD, Yamada Y, Fourney DR (2010) A novel classification system for spinal instability in neoplastic disease: an evidence-based approach and expert consensus from the Spine Oncology Study Group. Spine (Phila Pa 1976) 35:E12211229. https://doi.org/10.1097/BRS.0b013e3181e16ae2

6. Fourney DR, Frangou EM, Ryken TC, Dipaola CP, Shaffrey CI, Berven SH, Bilsky MH, Harrop JS, Fehlings MG, Boriani S, Chou D, Schmidt MH, Polly DW, Biagini R, Burch S, Dekutoski MB, Ganju A, Gerszten PC, Gokaslan ZL, Groff MW, Liebsch NJ, Mendel E, Okuno SH, Patel S, Rhines LD, Rose PS, Sciubba DM, Sundaresan N, Tomita K, Varga PP, Vialle LR, Vrionis FD, Yamada Y, Fisher CG (2011) Spinal instability neoplastic score: an analysis of reliability and validity from the spine oncology study group. J Clin Oncol 29:3072-3077. https://doi.org/10.1200/jco.2010.34.3897

7. Versteeg AL, van der Velden JM, Verkooijen HM, van Vulpen M, Oner FC, Fisher CG, Verlaan JJ (2016) The effect of introducing the spinal instability neoplastic score in routine clinical practice for patients with spinal metastases. Oncologist 21:95-101. https://doi. org/10.1634/theoncologist.2015-0266

8. Versteeg AL, Verlaan JJ, Sahgal A, Mendel E, Quraishi NA, Fourney DR, Fisher CG (2016) The Spinal Instability Neoplastic Score: Impact on Oncologic Decision-Making. Spine (Phila 1976) 41(Suppl 20):S231-s237. https://doi.org/10.1097/brs.0000000000 001822

9. Pithwa YK, Sinha Roy V (2021) Can we extrapolate SINS score to evaluate instability in spinal tuberculosis? Global Spine J. https:// doi.org/10.1177/21925682211030876

10. Landis JR, Koch GG (1977) The measurement of observer agreement for categorical data. Biometrics 33:159-174

11. White AA, Southwick WO, Panjabi MM (1976) Clinical instability in the lower cervical spine a review of past and current concepts. Spine 1:15-27

12. Fantoni M, Trecarichi EM, Rossi B, Mazzotta V, Di Giacomo G, Nasto LA, Di Meco E, Pola E (2012) Epidemiological and clinical features of pyogenic spondylodiscitis. Eur Rev Med Pharmacol Sci 16(Suppl 2):2-7

13. Fehlings MG, Cooper PR, Errico TJ (1994) Posterior plates in the management of cervical instability: long-term results in 44 patients. J Neurosurg 81:341-349. https://doi.org/10.3171/jns.1994.81.3.0341

14. Tomita K, Kawahara N, Kobayashi T, Yoshida A, Murakami H, Akamaru T (2001) Surgical strategy for spinal metastases. Spine (Phila Pa 1976) 26:298-306. https://doi.org/10.1097/00007632200102010-00016

15. Asdourian PL, Mardjetko S, Rauschning W, Jónsson H Jr, Hammerberg KW, Dewald RL (1990) An evaluation of spinal deformity in metastatic breast cancer. J Spinal Disord 3:119-134

16. Dimar JR 2nd, Voor MJ, Zhang YM, Glassman SD (1998) A human cadaver model for determination of pathologic fracture threshold resulting from tumorous destruction of the vertebral body. Spine 
(Phila Pa 1976) 23:1209-1214. https://doi.org/10.1097/00007632199806010-00006

17. Windhagen H, Hipp JA, Hayes WC (2000) Postfracture instability of vertebrae with simulated defects can be predicted from computed tomography data. Spine (Phila Pa) 25:1775-1781. https://doi.org/10. 1097/00007632-200007150-00007

18. Windhagen HJ, Hipp JA, Silva MJ, Lipson SJ, Hayes WC (1997) Predicting failure of thoracic vertebrae with simulated and actual metastatic defects. Clin Orthop Relat Res 344:313-319
Publisher's Note Springer Nature remains neutral with regard to jurisdictional claims in published maps and institutional affiliations. 